\title{
Sharp Thresholds for Monotone Non Boolean Functions and Social Choice Theory
}

\author{
Gil Kalai * $\quad$ Elchanan Mossel ${ }^{\dagger}$
}

December 14, 2017

\begin{abstract}
A key fact in the theory of Boolean functions $f:\{0,1\}^{n} \rightarrow\{0,1\}$ is that they often undergo sharp thresholds. For example: if the function $f:\{0,1\}^{n} \rightarrow\{0,1\}$ is monotone and symmetric under a transitive action with $\mathbf{E}_{p}[f]=\epsilon$ and $\mathbf{E}_{q}[f]=1-\epsilon$ then $q-p \rightarrow 0$ as $n \rightarrow \infty$. Here $\mathbf{E}_{p}$ denotes the product probability measure on $\{0,1\}^{n}$ where each coordinate takes the value 1 independently with probability $p$.

The fact that symmetric functions undergo sharp thresholds is important in the study of random graphs and constraint satisfaction problems as well as in social choice.

In this paper we prove sharp thresholds for monotone functions taking values in an arbitrary finite sets. We also provide examples of applications of the results to social choice and to random graph problems.

Among the applications is an analog for Condorcet's jury theorem and an indeterminacy result for a large class of social choice functions.
\end{abstract}

\section{Introduction}

\subsection{Sharp thresholds}

A key fact in the theory of Boolean functions is that monotone symmetric functions undergo sharp thresholds. This fact has fundamental significance in the study of constraint satisfaction problems, random graph processes and percolation and in social choice.

The results of [4] show that for every $0<\epsilon<1 / 2$ there exists a $C>0$ such that for all $n$ and all $f:\{0,1\}^{n} \rightarrow\{0,1\}$ which is monotone and symmetric (see definitions below) if $\mathbf{E}_{p}[f]=\epsilon$ and $\mathbf{E}_{q}[f]=1-\epsilon$ then $0<q-p<C(\log n)^{-1}$. This result implies in particular that "Every monotone graph property has a sharp threshold" as [4] is titled. It also implies that symmetric voting systems aggregate information well, see for example [5] and the examples provided in the current paper.

\footnotetext{
${ }^{*}$ Hebrew University of Jerusalem, Yale University, and Microsoft, Israel. Supported by ISF, and NSF awards

${ }^{\dagger}$ U.C. Berkeley and Weizmann Institute of Science. Supported by DMS 0548249 (CAREER) award, by DOD ONR grant N0014-07-1-05-06, by ISF grant 1300/08 and by a Minerva Grant
} 


\subsection{Notation and Main Results}

Let $A$ be a finite set. Let $X=A^{n}$. For $\sigma \in S(n)$, a permutation on $n$ elements and $x \in A^{n}$ we denote by $y=x_{\sigma}$ the vector satisfying $y_{i}=x_{\sigma(i)}$ for all $i$. For $\sigma \in S(A)$ we write $y=\sigma(x)$ for the vector satisfying $y_{i}=\sigma\left(x_{i}\right)$ for all $i$. For $a \in A$ and $x, y \in X$ we write $x \leq_{a} y$ if $\left\{i: x_{i}=a\right\} \subset\left\{i: y_{i}=a\right\}$ and for all $i$ such that $y_{i} \neq a$ it holds that $x_{i}=y_{i}$. In other words if $x \leq_{a} y$ then for all $i$ if $x_{i} \neq y_{i}$ then $y_{i}=a$. It is easy to see that $\leq_{a}$ defines a partial order on $X$.

We say that $f: X=A^{n} \rightarrow A$ is monotone if for all $a \in A$ and $x, y \in X$ such that $x \leq_{a} y$ it holds that $f(x)=a$ implies that $f(y)=a$. We say that $f$ is symmetric if there exists a transitive group $\Sigma \subset S(n)$ such that $f\left(x_{\sigma}\right)=f(x)$ for all $x \in X$ and $\sigma \in \Sigma$. We say that $f$ is fair if for all $\sigma \in S(A)$ and all $x \in X$ it holds that $f(\sigma(x))=\sigma(f(x))$.

Let $\Delta[A]$ denote the simplex of probability measures on $A$ and let $\gamma$ denote the standard probability measure on $\Delta[A]$. For $\mu \in \Delta[A]$ denote by $\mathbf{P}_{\mu}$ the measure $\mu^{\otimes n}$ on $X$. We denote by $\mathbf{E}_{\mu}$ the expected value according to the measure $\mathbf{P}_{\mu}$. For any measure $\mu \in \Delta[A]$, we write $\mu^{*}$ for the minimal probability $\mu$ assigns to any of the atoms in $A$.

In our main result we show that:

Theorem 1.1. There exists an absolute constant $C=C(|A|)$ such that if $f$ is symmetric and monotone then for any $a \in A$ and $\epsilon>0$ it holds that

$$
\gamma\left[\mu: \epsilon \leq P_{\mu}[f=a] \leq 1-\epsilon\right] \leq C(\log (1-\epsilon)-\log (\epsilon)) \frac{\log \log n}{\log n} .
$$

The result above establishes sharp threshold for symmetric functions as it shows that for almost all probability measures $f$ takes one specific value with probability at least $1-\epsilon$.

Remark 1.2. It is interesting to compare the results established here to those of [4]. For $|A|=2$ our results give a threshold interval of length $O(\log \log n / \log n)$ compared to the results of [4] which give threshold interval of length $O(1 / \log n)$. In the binary case the later result is tight. It is natural to conjecture that the threshold is always of measure $O(1 / \log n)$.

\subsubsection{Other applications}

Various applications of the threshold result to problems involving distributions of edge colored graphs are given in subsection 3.2.

\subsection{Social choice background, and applications}

We will describe two main applications of our main result to social choice theory. The first application gives an extension of Condorcet's Jury theorem for monotone choice functions for more than two candidates and large classes of voting rules. The second application is to indeterminacy results for generalized social choice functions. 


\subsubsection{Aggregation of Information}

The law of large numbers implies that in an election between two candidates denoted 0 and 1 , if every voter votes for 0 with probability $p>1 / 2$ and for 1 with probability $1-p$ and if these votes are independent, then as the number of voters tends to infinity the probability that 0 will be elected tends to one. This fact is referred to as Condorcet's Jury Theorem.

This theorem can be interpreted as saying that even if agents receive very poor but independent signals indicating which decision is correct, majority voting will nevertheless result in the correct decision being taken with a high probability if there are enough agents (and each agent votes according to the signal he receives). This phenomenon is referred to as asymptotically complete aggregation of information and it plays an important role in theoretical economics.

More recent results studied aggregation of information for general symmetric fair functions $f:\{0,1\}^{n} \rightarrow\{0,1\}$. Recall that such a function is fair if $f\left(1-x_{1}, \ldots, 1-x_{n}\right)=1-f\left(x_{1}, \ldots, x_{n}\right)$. In this setup the results of [4] imply that for every $p>1 / 2$ and every symmetric fair function on $n$ voters it holds $\mathbf{E}_{p}[f]>1-o(1)$.

Here we derive a similar result in the case of an election between $[q]$ candidates. Note that the conditions of monotonicity and fairness are both natural in this setup.

- Monotonicity implies that if in a certain vector $x \in[q]^{n}$ of voters a certain candidate $i$ is elected and if $y$ is identical to $x$ except that some of the voters changed their mind as to vote $i$, then the outcome of the vote for $y$ should also be $i$.

- Fairness means that all the candidates are treated equally.

Theorem 1.3. For every $q$ there exists a constant $C=C(q)$ for which the following holds for every $\epsilon<1 / 3$. Let $\mu \in \Delta(q)$ and $i \in[q]$ satisfy that

$$
\mu(i)>\max _{j \neq i} \mu(j)+C(\log (1-\epsilon)-\log (1 / q)) \frac{\log \log n}{\log n} .
$$

Then for every fair monotone function $f:[q]^{n} \rightarrow[q]$ it holds that

$$
\mu[f=i] \geq 1-\epsilon .
$$

In words - the proposition claims that for any measure on the votes that has a $\log \log n / \log n$ bias towards one of the candidates $i$ and any fair monotone voting function it holds that $i$ will be elected with high probability. The proof is given in subsection 3.1.

\subsubsection{Indeterminacy}

Arrow's impossibility theorem asserts that under certain natural conditions, if there are at least three alternatives then every non-dictatorial social choice gives rise to a non-rational choice function, i.e., there exist profiles such that the social choice is not rational. Arrow's theorem can be seen in the context of Condorcet's "paradox" which demonstrates that the majority rule may result in the society preferring A over B, B over C and C over A. Arrow's theorem shows that such 
"paradoxes" cannot be avoided with any non-dictatorial voting method. It is the general form of Arrow's theorem, which can be applied to general schemes for aggregating individual rational choices, that made it so important in economic theory.

McGarvey [7] appears to have been the first to show that for every asymmetric relation $R$ on a finite set of candidates there is a strict-preferences (linear orders, no ties) voter profile that has the relation $R$ as its strict simple majority relation. This implies that we cannot deduce the society's choice between two candidates even if we know the society's choice between every other pair of candidates. We refer to this phenomena as "complete indeterminacy".

Saari [9] proved that the plurality method gives rise to every choice function for sufficiently large societies. (In fact, he proved more: the ranking on any subset of the alternatives can be prescribed). This implies that knowing the outcome of the plurality choice for several examples, where each example consists of a set $S$ of alternatives and the chosen element $c(S)$ for $S$, cannot teach us anything about the outcome for a set of alternatives which is not among the examples we have already seen.

In [5] McGarvey's theorem is extended to sequences of neutral social welfare functions in which the maximum Shapley-Shubik power index tends to 0. The proof relied on threshold properties of Boolean functions. In particular, McGarvey's theorem extends to neutral social welfare functions which are invariant under a transitive group of permutations of the individuals. We describe a similar extension of Saari's theorem.

\section{Proof of the main theorem}

The proof follows the same simple idea used in [4]. That is, we use information on the influences in order to deduce that $\mathbf{E}_{\mu}[f]$ varies quickly as a function of $\mu$. We thus derive a generalization of Russo's formula $[6,8]$ which expresses derivatives in terms of influences. We then use the fact that functions taking only a bounded number of values must have large influence sums.

Depending on the type of influence sum bounds, one obtain different results. Using the results of [1] it is possible to obtain a bound on the threshold interval length of order $O\left(\log ^{1 / 3} n\right)$. Here we derive the better bound of order $O(\log \log n / \log n)$ using a generalization of the results of Talagrand [10]. This generalization was proven in a course taught by the second author on Fall 2005. A draft of the proof was written in scribe notes of the course by Asaf Nachmias at http : //www.stat.berkeley.edu/ mossel/teach/206af05/scribes/oct25.pdf. For completeness we have corrected and completed the proof.

It seems like in order to obtain a threshold bound of $O(1 / \log n)$ it would be needed to derive a tighter influence sum bound tailored to the setup here.

From now on, without loss of generality assume $A=[q]=\{0,1 \ldots, q-1\}$. We will consider functions $f:[q]^{n} \rightarrow\{0,1\}$ and say that such a function is 0 -monotone if $x \leq_{0} y$ implies that $f(x) \leq f(y)$.

Clearly in order to prove Theorem 1.1, it suffices to prove that there exists $C>0$ such that if 
$f$ is symmetric and 0-monotone then for all $\epsilon>0$ it holds that

$$
\gamma\left[\mu: \epsilon \leq P_{\mu}[f=0] \leq 1-\epsilon\right] \leq C(\log (1-\epsilon)-\log (\epsilon)) \frac{\log \log n}{\log n} .
$$

\section{$2.1 \quad$ Influences}

We will use the $L_{2}$ influence

Let $f: X \rightarrow \mathbb{R}$.

Let $I_{\mu}^{i}(f)$, the $i$ 'th influence of $f$ according to $\mu \in \Delta[A]$ by

$$
I_{\mu}^{i}(f)=\mathbf{E}_{\mu}\left[\operatorname{Var}\left[f \mid x_{1}, \ldots, x_{i-1}, x_{i+1}, \ldots, x_{n}\right]\right]
$$

In Corollary 5.7 we will derive the following lower bound on influence sum which is a consequence of a generalization of a result of Talagrand [10]. We restate the corollary here:

Corollary 2.1. There exists some universal constant $C$ such that for any probability space $(\Omega, \mu)$ and any function $f: \Omega^{n} \rightarrow\{0,1\}$ which is symmetric it holds that it holds that

$$
\sum_{i=1}^{n} I_{i}(f) \geq \frac{C}{\log (1 / \alpha)} \log n \operatorname{Var}(f)
$$

\subsection{Russo Type Formula}

We denote by $T(\Delta)$ the tangent space to $\Sigma(\Delta)$. The space is easily identified with the space of all vectors in $t \in \mathbb{R}^{A}$ satisfying $\sum t_{i}=0$. The natural derivative on $T(\Delta)$ satisfies $\partial P_{\mu}[f] / \partial t \in T(\Delta)$.

Lemma 2.2. Let $f:[q] \rightarrow\{0,1\}$ be a 0 -monotone function and $\mu$ a measure on $[q]$ and suppose that $f$ is 0-monotone. Write $\mu=(1-\mu(0)) \mu^{\prime}+\mu(0) \delta_{0}$ where $\mu^{\prime}(0)=0$ and $\mu^{\prime}$ is a probability measure. Let $t=\delta_{0}-\mu^{\prime} \in T(\Delta)$. Then

$$
\frac{\partial \mathbf{E}_{\mu+h t}[f]}{\partial h}=1(f \text { is not constant }) \mu^{\prime}[1-f] \geq \operatorname{Var}_{\mu^{\prime}}[f] \text {. }
$$

Proof. By linearity the derivative equals $f(0)-\mu^{\prime}[f]$. The last expression is 0 when $f$ is constant. If $f$ is not constant then by monotonicity it holds that $f(0)=1$ and therefore the derivative is $1-\mu^{\prime}[f]$ as needed. Finally, the inequality follows from the fact that if $f$ is constant then the variance is 0 and otherwise $\mu^{\prime}[1-f] \geq \mu^{\prime}[f] \mu^{\prime}[1-f]=\operatorname{Var}_{\mu^{\prime}}[f]$.

We now prove a generalization of Russo's formula.

Lemma 2.3. Let $f:[q]^{n} \rightarrow\{0,1\}$ be a 0-monotone function and $\mu \in \Delta[q]$. Write $\mu=(1-$ $\mu(0)) \mu^{\prime}+\mu(0) \delta_{0}$ where $\mu^{\prime}(0)=0$ and $\mu^{\prime}$ is a probability measure. Let $t=\delta_{0}-\mu^{\prime} \in T(\Delta)$. Then:

$$
{\frac{\partial \mathbf{E}_{\mu+h t}[f]}{\partial h}}_{\mid h=0}=\sum_{i=1}^{n} \mathbf{E}_{\mu}\left[1\left(\left[f \mid F_{i}\right] \text { is not constant }\right) \mathbf{E}_{\mu^{\prime}}\left[1-f \mid F_{i}\right]\right] \geq \sum_{i=1}^{n} I_{\mu^{\prime}}^{i}(f),
$$

where $F_{i}$ denotes conditioning on $x_{1}, \ldots, x_{i-1}, x_{i+1}, \ldots, x_{n}$. 
Proof. Use the chain rule:

$$
{\frac{\partial \mathbf{E}_{\mu+h t}[f]}{\partial h} \mid h=0}_{\left|h={\frac{\partial(\mu+h t)^{\otimes n}[f]}{\partial h}}_{\mid h=0}=\sum_{i=1}^{n} \frac{\partial \otimes_{j=1}^{n}\left(\mu+h_{j} t\right)[f]}{\partial h_{i}}\right|\left(h_{1}, \ldots, h_{n}\right)=0}
$$

and Lemma 2.2.

\subsection{Proof of the Main Result}

We now prove the main result, i.e., (1). In the proof below the constants $c$ will denote different constant at different lines. All of them depend on $q$ only.

Proof. Let $\Gamma$ denote the set of measures $\mu$ in $\Delta[q]$ satisfying the following:

- $\mu[0]=0$.

- $\mu[i] \geq \eta$ for all $i \neq 0$.

Write $\mu^{t}=t \delta_{0}+(1-t) \mu$ and note that

$$
\gamma\left[\mu^{t}: 0 \leq t \leq 1, \mu \notin \Gamma\right] \leq C(q) \eta .
$$

For each measure $\mu \in \Gamma$ we look at the interval $J_{\mu}^{\epsilon, 1-\epsilon}$, where

$$
J_{\mu}^{\epsilon, 1-\epsilon}=\left\{t \in I_{\mu}: \epsilon \leq P_{\mu^{t}}[f] \leq 1-\epsilon\right\} .
$$

Our goal is to show that the interval $J_{\mu}^{\epsilon, 1-\epsilon}$ is short by bounding the derivative of

$$
G(t)=\mu^{t}[f]
$$

in the interval. In fact we will bound the length of each of the sub-intervals $J_{\mu}^{\epsilon, 1 / 2}$ and $J_{\mu}^{1 / 2,1-\epsilon}$. Since $f$ is 0,1 valued, it follows that in the interval $J_{\mu}^{\epsilon, 1 / 2}$ it holds that $\operatorname{Var}_{\mu^{t}}[f] \geq \frac{1}{2} \mu^{t}[f]$. By Lemma 2.3 and Proposition 2.1 we conclude that:

$$
G^{\prime}(t) \geq \sum_{i=1}^{n} I_{\mu^{t}}^{i}(f) \geq \frac{c \log n}{\log (1 / \eta)} \operatorname{Var}_{\mu}[f] \geq \frac{c \log n}{2 \log (1 / \eta)} G(t) .
$$

This implies that:

$$
(\ln G)^{\prime}(t) \geq \frac{c \log n}{\log (1 / \eta)}
$$

In particular if $G(p)=\epsilon$ and $G(q)=1 / 2$ then:

$$
\ln (1 / 2)-\ln (\epsilon) \geq \frac{c|p-q| \log n}{\log (1 / \eta)}
$$

So:

$$
|p-q| \leq \frac{(\ln (1 / 2)-\ln (\epsilon)) \log (1 / \eta)}{c \log n} .
$$


Repeating the same argument for the interval $(1 / 2,1-\epsilon)$ we obtain that

$$
\left|J_{\mu}^{\epsilon, 1-\epsilon}\right| \leq \frac{(\ln (1-\epsilon)-\ln (\epsilon)) \log (1 / \eta)}{c \log n} .
$$

Which together (2) with implies that

$$
\gamma\left[\mu: \epsilon \leq P_{\mu}[f=0] \leq 1-\epsilon\right] \leq C(q)\left(\eta+\frac{(\ln (1-\epsilon)-\ln (\epsilon)) \log (1 / \eta)}{\log n}\right) .
$$

Taking $\eta=(\ln (1-\epsilon)-\ln (\epsilon)) / \log n$ we obtain the bound:

$$
C(q)\left(\frac{\ln (1-\epsilon)-\ln (\epsilon)}{\log n}+(\ln (1-\epsilon)-\ln (\epsilon)) \frac{\log \log n}{\log n}\right) \leq 2 C(q)\left((\ln (1-\epsilon)-\ln (\epsilon)) \frac{\log \log n}{\log n}\right.
$$

as needed.

\section{Applications}

\subsection{A general form of Condorcet's Jury theorem}

We begin with a proof of Theorem 1.3. Since the proof is similar to the previous proof, we only sketch the main steps.

Proof. We only sketch the proof since it is similar to the proof of Theorem 1.1. Assume first that $\mu(j)>\frac{1}{\log n}$ for all $j$. Let $\mu=t^{*} \delta_{i}+\left(1-t^{*}\right) \mu^{\prime}$ where $\mu^{\prime}$ is a probability measure with $\mu^{\prime}(i)=0$. As before write $\mu^{t}=t \delta_{i}+(1-t) \mu^{\prime}$ for every $t$. Let $s<t^{*}$ be chosen so that $\mu^{s}(i)=\max _{j \neq i} \mu^{s}(j)$. From symmetry and monotonicity it follows that

$$
\mu^{s}[f=i] \geq 1 / q
$$

Moreover, the argument in Theorem 1.1 shows that for $t$ in the interval $\left(s, t^{*}\right)$ it holds that

$$
{\frac{\partial \mu^{t+h}[f=i]}{\partial h}}_{\mid h=0} \geq C \mu^{t}[f=i]\left(1-\mu^{t}[f=i]\right) \frac{\log \log n}{\log n},
$$

which implies that

$$
\left|t^{*}-s\right| \leq C(\log (1-\epsilon)-\log (1 / q)) \frac{\log \log n}{\log n}
$$

thus proving the statement of the theorem.

It remains to remove the assumption that $\mu(j)>\frac{1}{\log n}$ for all $j$. The general case where some of the probabilities $\mu(i)$ may satisfy $\mu(i)<\frac{1}{\log n}$ requires one additional step at the cost of taking the constant $C$ to be $C^{\prime}=C+(q-1)$. Instead of the measure $\mu$ we first consider the measure $\hat{\mu}$ where $\hat{\mu}(j)=\mu(j)+1 / \log n$ for $j \neq i$ and $\hat{\mu}(i)=\mu(i)-(q-1) / \log n$. 
Note that if $\mu$ satisfies the conditions of the theorem with the constant $C^{\prime}$ then $\hat{\mu}$ satisfies it with the constant $C$. Moreover $\hat{\mu}(j)>1 / \log n$ for all $j$. Therefore by the first part of the proof

$$
\hat{\mu}[f=i] \geq 1-\epsilon .
$$

On the other hand, by monotonicity we have

$$
\mu[f=i] \geq \hat{\mu}[f=i],
$$

which implies the desired result.

\subsection{Graph properties}

Consider a process on the edges of the complete graph $K_{n}$ where each edge $e=\{a, b\}$ is labeled by $i$ with probability $\mu(i)$ for $1 \leq i \leq q$ independently for different edges. This process defines sets $E_{1}, \ldots, E_{q}$ where $E_{i}$ is the set of edges labeled by $i$. In other words, it defines $q$ graphs $\left([n], E_{1}\right), \ldots,\left([n], E_{q}\right)$.

Definition 3.1. A function $A$ from the set of partitions of the edges of $K_{n}$ to $q$ parts into $[q]$ is called a graph property if: For all partitions $E_{1}, \ldots, E_{q}$ and all permutations $\sigma \in S(n)$ it holds that

$$
A\left(E_{1}, \ldots, E_{q}\right)=A\left(\sigma\left(E_{1}\right), \ldots, \sigma\left(E_{q}\right)\right),
$$

where $\sigma\left(E_{i}\right)=\left\{\{\sigma(u), \sigma(v)\}:\{u, v\} \in E_{i}\right\}$.

The function $A$ is called a monotone graph property if

- For every pair of partitions $\left(E_{1}, \ldots, E_{q}\right)$ and $\left(F_{1}, \ldots, F_{q}\right)$

- and all $1 \leq i \leq q$ it holds that

If:

- $E_{i} \subseteq F_{i}$ and

- $F_{j} \subseteq E_{j}$ for $j \neq i$ and

- $A\left(E_{1}, \ldots, E_{q}\right)=i$,

Then $A\left(F_{1}, \ldots, F_{q}\right)=i$.

Here are a few examples:

- Given a graph labeled by $[q]$, let $A\left(E_{1}, \ldots, E_{q}\right)=i$, where $i$ is the minimal index for which $\left|E_{i}\right|$ is maximal. In other words, $i$ is the most popular label, where ties are decided by preferring the smaller index.

- Given a graph labeled by $[q]$ let $A\left(E_{1}, \ldots, E_{q}\right)=i$ where $E_{i}$ has the largest clique. Again ties are decided by preferring the smaller index. 
- Given a graph labeled by $[q]$ let $A\left(E_{1}, \ldots, E_{q}\right)=i$ where $E_{i}$ has the smallest independent set of vertices. Again ties are decided by preferring the smaller index.

Theorem 1.1 implies the following:

Corollary 3.2. There exists a constant $C=C(q)$ such that for every monotone graph property it holds that

$$
\gamma\left[\mu: \epsilon \leq P_{\mu}[A=i] \leq 1-\epsilon\right] \leq C(\log (1-\epsilon)-\log (\epsilon)) \frac{\log \log n}{\log n} .
$$

\section{Indeterminacy for voting methods}

\subsection{The setting}

Let $P_{+}(X)$ denote the family of non-empty subsets of $X$.

Definition 4.1. Given a set $X$ of $m$ alternatives, a choice function $c$ is a mapping which assigns to each nonempty subset $S$ of $X$ an element $c(S) \in S$. A choice function is thus a map $c: P_{+}(X) \rightarrow X$ with the additional property that $c(S) \in S$ for all $S \in P_{+}(X)$.

A choice function is called rational if there is a linear ordering on the alternatives such that $c(S)$ is the maximal element of $S$ according to that ordering.

A social choice function is a map

of the form $c=F\left(c_{1}, c_{2}, \ldots, c_{n}\right)$ where $c$ is a choice function on $X$ which depends on the profile of individual rational choice functions $c_{1}, c_{2}, \ldots c_{n}$ for the individuals.

Note that there are two different meanings for the term "social choice functions". Sometimes a social choice function is referred to as a map which associates to the profile of rational individual choices (or preferences) a single "winner" for the society. A social choice function in this sense easily defines a social choice function in our sense by restricting to a subset of alternatives. We can regard social choice functions as election rules which given a set $S$ of candidates and (strict) preference relations of the individuals on the candidates, provides a rule for choosing the winner. We regard $X$ as the set of all possible candidates, and $S$ as the set of available candidates, and we are interested to understand the society's choice as a function of $S$.

The axiom of Independence of Irrelevant Alternatives (Arrow's IIA) asserts that $c(A)$ may depend only on the preference relations restricted to the set $A$.

We require the stronger property Independence of Rejected Alternatives (IRA), also referred to as Nash's IIA.

(IRA) (Independence of Rejected Alternative (IRA)) $c(S)$ is a function of $\left(c_{1}(S), c_{2}(S), \ldots c_{n}(S)\right)$

Therefore we can write $c(S)=F_{S}\left(c_{1}(S), c_{2}(S), \ldots c_{m}(S)\right)$.

We will require a few more conditions:

(P) (Pareto) $c(S) \in\left\{c_{1}(S), c_{2}(S), \ldots, c_{n}(S)\right\}$. 


\subsection{Further assumptions}

We will make the following additional assumptions:

(U) (Unrestricted domains) The social choice function is defined for arbitrary rational profiles of the individuals.

(N1') (Neutrality) The social choice is invariant under permutations of the alternatives.

(A2') (Weak anonymity) The social choice is invariant under a transitive group of permutations of the individuals.

(M') (Monotonicity) The function $c(S)$ is monotone in the following sense: If $c(S)=s$ and $c_{i}(S)=t$ for $t \neq s$ then changing the choice of the $i$-th individual from $t$ to $s$ will not change $c(S)$.

\subsection{The result}

Theorem 4.1. Let $c_{0}$ be an arbitrary choice function on $P_{+}(X)$ where $X$ is a set of $m$ alternatives and let $\delta<1$.

Then there is a probability distribution $\nu=\nu\left(c_{0}\right)$ on the space of orderings of the alternatives and a number $N=N(\delta, m)$ such that the following holds for every social choice function $F$ which satisfies conditions (IRA) (P) (U') (N1'), (A2') and (M'):

If the number of individuals $n$ is larger than $N$ and if every individual makes the choice randomly and independently according to $\nu$, then with probability at least $\delta$ for all $S \in P_{+}(X)$ it holds that $c=F\left(c_{1}, \ldots, c_{n}\right)$ satisfies $c(S)=c_{0}(S)$ with probability of at least $\delta$ (here $c_{i}(S)$ is the highest ranked alternative in the order of voter $i)$.

Corollary 4.2. There exists $N=N(m)$ such that when the number $n$ of individuals is larger than $N(m)$, every choice function $c$ on $P_{+}(X)$, where $X$ is a set of $m$ alternatives, can be written as $c=F\left(c_{1}, \ldots, c_{n}\right)$ for every social choice function $F$ satisfies conditions (IRA), (P), (U') (N1'), (A2') and ( $\left.M^{\prime}\right)$ applied to $c_{1}, \ldots, c_{n}$ that are determined by rankings.

\subsection{The proof of Theorem 4.1}

Proof. Let $c_{0}$ be an arbitrary choice function and consider a profile with $n_{0}$ individuals such that the plurality leads to $c_{0}$. Such a profile exists by Saari's theorem.

For an ordering $\pi$ of the alternatives let $w^{\prime}(\pi)$ be the number of appearances of the order $\pi$ and let $w(\pi)=w^{\prime}(\pi) / n_{0}$.

Consider a random profile $\left(c_{1}, \ldots, c_{n}\right)$ on $n$ individuals where for each individual $i$ the probability that $i$-th preference relation $c_{i}$ is described by $\pi$ is $w(\pi)$ (independently for the individuals).

We will show that for all $\delta^{\prime}<1$ if $n=n\left(\delta^{\prime}\right)$ is sufficiently large, then for $S \subset X$ it holds with probability at least $\delta^{\prime}$ that $c(S)=F_{S}\left(c_{1}(S), \ldots, c_{n}(S)\right)$ satisfies $c(S)=c_{0}(S)$ with probability at least $\delta^{\prime}$. This implies the required result by taking $1-\delta^{\prime} \leq 2^{-m}(1-\delta)$. 
To establish the claim above note that $c_{1}(S), \ldots, c_{n}(S)$ are i.i.d. and that for all $a \neq c_{0}(S)$ it holds that $P\left[c_{i}(S)=c_{0}(S)\right] \geq P\left[c_{i}(S)=a\right]+n_{0}^{-1}$. Furthermore the function $F_{S}$ as a functions of $c_{1}(S), \ldots, c_{n}(S)$ satisfies neutrality and weak anonymity. Thus from Theorem 1.3 it follows that for $n$ large enough the probability that $F_{S}\left(c_{1}(S), \ldots, c_{n}(S)\right)=c_{0}(S)$ is at least $1-\delta^{\prime}$ as needed.

Note that the proof actually implies the following in the spirit of a theorem by Dasgupta and Maskin [2].

Corollary 4.3. There exists $N=N(m)>0$ with the following property: Let $U$ be a set of linear orders on $m$ alternatives. If for the plurality rule there is a profile restricted to $U$ which leads to a choice function $c$ for the society, then when $n>N(m)$ this is the case for every social choice function which satisfies conditions (IRA), (P), (N1'), (A2') and (M').

\subsection{Taking rejected alternatives into account}

We now describe a crucial example suggested by Bezalel Peleg. First, we note how we can base on choices on pairs a choice correspondence: Given an asymmetric binary relation $R$ on the set of alternatives let $c(S)$ be the set of elements $y$ of $S$ such that the number of $z \in S$ such that $y R z$ is maximal. In other words, when we consider the directed graph described by the relation we choose the vertex of maximal out-degree.

Let $\mathcal{R}$ denote the class of rational choice functions and $\mathcal{B}$ denote the class of choice correspondences obtained from binary relations $R$ as just described. Consider also the class $\mathcal{B}^{\prime}$ of choice functions obtained from $\mathcal{B}$ by choosing a single element in $c(S)$ according to some fixed order relation on the alternatives. The number of choice functions in $\mathcal{B}^{\prime}$ and the number of choice correspondences in $\mathcal{B}$ is exponential in $\left(\begin{array}{l}n \\ 2\end{array}\right)$.

Now describe a social choice function as follows: $a R b$ if a majority of the society prefers $a$ to $b$.

In this case the social choice of $S$ does not depend solely on the individual choices for $S$ but also on the preferences among pairs of elements in $S$.

When the individual choices are rational then the social choice still belongs to the class $\mathcal{B}$ (or $\left.\mathcal{B}^{\prime}\right)$. In this case the choice from $S$ is simply those elements of $S$ which are Condorcet winners against the maximal number of other elements in $S$. In this example the social choice for a set $S$ is typically large but this apparently be corrected by various methods of "tie breaking".

In these examples the size of the resulting classes of choice functions is exponential in a quadratic function of $m^{2}$. It is much smaller than the number of all choice functions which is double exponential in $m$.

The Borda rule can be analyzed by a similar consideration.For this rule $c(A)$ is determined as follows: For each alternative $a \in A$ let $r_{i}(a)$ be the number of individuals who ranked $a$ in the $i$ th place (among the elements of $A$ ). Let $r(a)=\sum i \cdot r_{i}(a)$. The chosen element by the society $c(A)$ is the element of $a$ with the minimal weight.

Another way to describe the Borda rule is as follows: First construct a directed graph (with multiple edges) with $A$ as the set of vertices by introducing an edge from $a$ to $b$ for every individual 
that prefers $a$ to $b$. Next, define (as before) $c(A)$ as the vertex with maximal outdegree.

It is easy to prove that the number of choice functions that arise in this way is at most exponential in $m^{3}$. (The choice function can be recovered from the sign patters of (less than) $2^{m} \cdot m^{2}$ linear expressions in $m^{2}$ real variables.

To summarize, the size of classes of choice functions that arise from a social choice function such that $c(S)$ may depend on the individual preferences of the elements of $S$ is at least exponential in $m^{2}$ and this bound is sharp.

\section{A generalization of Talagrand's result}

In this section we prove the bounds on $L^{2}$ influence sums for arbitrary probability spaces. For this we first recall the notion of Efron-Stein decomposition [3] then generalize Talagrand's result [10] and finally derive corollaries for $\{0,1\}$-valued and symmetric functions.

\subsection{Efron-Stein decomposition}

Consider finite probability spaces $\Omega_{1}, \ldots, \Omega_{n}$, with measures $\mu_{1}, \ldots, \mu_{n}$. Let $\alpha_{i}$ be size of the smallest atom of $\left(\Omega_{i}, \mu_{i}\right)$, and set $\alpha=\min _{i} \alpha_{i}$. Let $f \in L^{2}\left(\prod_{i} \mu_{i}\right)$ be a real valued function. Write $f=\sum_{S \subset[n]} f_{S}$ for the Efron-Stein decomposition of $f$ which we now recall.

Definition 5.1. Let $\left(\Omega_{1}, \mu_{1}\right), \ldots,\left(\Omega_{n}, \mu_{n}\right)$ be discrete probability spaces $(\Omega, \mu)=\prod_{i=1}^{n}\left(\Omega_{i}, \mu_{i}\right)$. The Efron-Stein decomposition of $f: \Omega \rightarrow \mathbb{R}$ is given by

$$
f(x)=\sum_{S \subseteq[n]} f_{S}\left(x_{S}\right),
$$

where the functions $f_{S}$ satisfy:

- $f_{S}$ depends only on $x_{S}$.

- For all $S \nsubseteq S^{\prime}$ and all $x_{S^{\prime}}$ it holds that:

$$
\mathbf{E}\left[f_{S} \mid X_{S^{\prime}}=x_{S^{\prime}}\right]=0
$$

It is well known that the Efron-Stein decomposition exists and that it is unique [3]. We quickly recall the proof of existence. The function $f_{S}$ is given by:

$$
f_{S}(x)=\sum_{S^{\prime} \subseteq S}(-1)^{\left|S \backslash S^{\prime}\right|} \mathbf{E}\left[f(X) \mid X_{S^{\prime}}=x_{S^{\prime}}\right]
$$

which implies

$$
\sum_{S} f_{S}(x)=\sum_{S^{\prime}} \mathbf{E}\left[f \mid X_{S^{\prime}}=x\right] \sum_{S: S^{\prime} \subseteq S}(-1)^{\left|S \backslash S^{\prime}\right|}=\mathbf{E}\left[f \mid X_{[n]}=x_{[n]}\right]=f(x) .
$$


Moreover, for $S \nsubseteq S^{\prime}$ we have $\mathbf{E}\left[f_{S} \mid X_{S^{\prime}}=x_{S^{\prime}}\right]=\mathbf{E}\left[f_{S} \mid X_{S^{\prime} \cap S}=x_{S^{\prime} \cap S}\right]$ and for $S^{\prime}$ that is a strict subset of $S$ we have:

$$
\begin{aligned}
\mathbf{E}\left[f_{S} \mid X_{S^{\prime}}=x_{S^{\prime}}\right] & =\sum_{S^{\prime \prime} \subset S}(-1)^{\left|S \backslash S^{\prime \prime}\right|} \mathbf{E}\left[f(X) \mid X_{S^{\prime \prime} \cap S^{\prime}}=x_{S^{\prime \prime} \cap S^{\prime}}\right] \\
& =\sum_{S^{\prime \prime} \subset S^{\prime}} \mathbf{E}\left[f(X) \mid X_{S^{\prime \prime}}=x_{S^{\prime \prime}}\right] \sum_{S^{\prime \prime} \subset \tilde{S} \subset S^{\prime \prime} \cup\left(S \backslash S^{\prime}\right)}(-1)^{|S \backslash \tilde{S}|}=0 .
\end{aligned}
$$

\subsection{Generalization of a Result of Talagrand}

We now prove:

Theorem 5.2 (Generalization of Talagrand, 1994). There exists some universal constant $C$ such that for any probability spaces $(\Omega, \mu)$ and any function $f \in L^{2}\left(\Omega^{n}, \mu^{n}\right)$ it holds that

$$
\operatorname{Var}(f) \leq C \log \left(1 / \mu^{*}\right) \sum_{i \leq n} \frac{\left\|\Delta_{i} f\right\|_{2}^{2}}{\log \left(\left\|\Delta_{i} f\right\|_{2} /\left\|\Delta_{i} f\right\|_{1}\right)}
$$

where $\Delta_{i} f=\sum_{S: i \in S} f_{S}$.

The proof is almost identical to Talagrand's proof using Efron-Stein decomposition instead of Fourier expansion and known bounds on the hyper-contractive constants of finite probability spaces. In particular we'll use the following result of Wolff [11]:

Theorem 5.3. For $g: \Omega^{n} \rightarrow \mathbb{R}$, let:

$$
T_{\Theta} g=\sum_{S} \Theta^{|S|} g_{S}
$$

Then for all $g$ it holds that:

$$
\left\|T_{\sigma} g\right\|_{2} \leq\|g\|_{3 / 2}
$$

for all

$$
\sigma \leq\left(\frac{(1-\alpha)^{2-4 / 3}-\alpha^{2-4 / 3}}{(1-\alpha) \alpha^{1-4 / 3}-\alpha(1-\alpha)^{1-4 / 3}}\right)^{1 / 2}
$$

Remark 5.4. In particular one may take $\sigma=\alpha^{2} / 6$.

Proof. Let

$$
f(x)=x^{2 / 3}, \quad g(x)=-(1-x) / x^{1 / 3} .
$$

By Lagrange's theorem we may take:

$$
\sigma^{2}=\frac{f^{\prime}\left(\xi_{1}\right)}{g^{\prime}\left(\xi_{2}\right)}
$$

for some $\xi_{1}, \xi_{2} \in(\alpha, 1-\alpha)$. Clearly

$$
f^{\prime}(x)=(2 / 3) x^{-1 / 3}, \quad g^{\prime}(x)=(2 / 3) x^{-1 / 3}+(1 / 3) x^{-4 / 3},
$$


are decreasing, and therefore

$$
\sigma^{2} \geq \frac{f^{\prime}(1-\alpha)}{g^{\prime}(\alpha)}=\frac{2(1-\alpha)^{-1 / 3}}{2 \alpha^{-1 / 3}+\alpha^{-4 / 3}} \geq \frac{2}{3 \alpha^{-4 / 3}} \geq \frac{\alpha^{2}}{6} .
$$

We now prove Theorem 2.1.

Proof. For a real function $g$ from our space, denote

$$
M^{2}(g)=\sum_{S \neq \emptyset} \frac{\left\|g_{S}\right\|_{2}^{2}}{|S|}
$$

So

$$
\operatorname{Var}[f]=\sum_{S \neq \emptyset}\left\|f_{S}\right\|_{2}^{2}=\sum_{i=1}^{n} M^{2}\left(\Delta_{i} f\right)
$$

Note that the statement of the theorem follows if we prove that for any function $g$ with $\mathbf{E} g=0$,

$$
M^{2}(g) \leq K \log (1 / \alpha) \frac{\|g\|_{2}^{2}}{\log \left(\|g\|_{2} /\|g\|_{1}\right)}
$$

The statement of the theorem follows by apply (4) to $\Delta_{i} f$ and summing the inequalities. To prove (4) we use hypercontractivity. The following proposition is proved in the end of this note.

Applying Theorem 5.3 gives that for any integer $k>0$,

$$
\sigma^{2 k} \sum_{0<|S|=k}\left\|g_{S}\right\|_{2}^{2} \leq \sum_{S} \sigma^{2|S|}\left\|g_{S}\right\|_{2}^{2}=\left\|T_{\sigma} g\right\|_{2}^{2} \leq\|g\|_{3 / 2}^{2},
$$

hence

$$
\sum_{|S|=k}\left\|g_{S}\right\|_{2}^{2} \leq\left(\frac{6}{\alpha^{2}}\right)^{k}\|g\|_{3 / 2}^{2} .
$$

Fix an integer $m>0$, and sum the previous inequality for all $k \leq m$ to get

$$
\sum_{|S| \leq m} \frac{\left\|g_{S}\right\|_{2}^{2}}{|S|} \leq \sum_{k \leq m} \frac{\left(\frac{6}{\alpha^{2}}\right)^{k}}{k}\|g\|_{3 / 2}^{2} \leq \frac{2\left(\frac{6}{\alpha^{2}}\right)^{m}}{m}\|g\|_{3 / 2}^{2},
$$

where the last inequality comes from the fact that the ratio between two consecutive summands in the sum is greater than 2. We now have

$$
\begin{aligned}
M^{2}(g) & =\sum_{|S| \leq m} \frac{\left\|g_{S}\right\|_{2}^{2}}{|S|}+\sum_{|S|>m} \frac{\left\|g_{S}\right\|_{2}^{2}}{|S|} \leq \frac{2\left(\frac{6}{\alpha^{2}}\right)^{m}}{m}\|g\|_{3 / 2}^{2}+\frac{\|g\|_{2}^{2}}{m} \\
& \leq \frac{2}{m}\left[\left(\frac{6}{\alpha^{2}}\right)^{m}\|g\|_{3 / 2}^{2}+\|g\|_{2}^{2}\right] .
\end{aligned}
$$


We now choose optimal $m$. Choose largest $m$ such that $\left(\frac{6}{\alpha^{2}}\right)^{m}\|g\|_{3 / 2}^{2} \leq\|g\|_{2}^{2}$, hence

$$
\left(\frac{6}{\alpha^{2}}\right)^{m+1}\|g\|_{3 / 2}^{2} \geq\|g\|_{2}^{2} \Longrightarrow m+1 \geq \frac{2 \log \left(\|g\|_{2} /\|g\|_{3 / 2}\right)}{\log \left(6 / \alpha^{2}\right)} .
$$

Plugging this back into (5) gives

$$
M^{2}(g) \leq C \frac{\log \left(6 / \alpha^{2}\right)\|g\|_{2}^{2}}{\log \left(\|g\|_{2} /\|g\|_{3 / 2}\right)} .
$$

An application of Cauchy-Schwartz gives

$$
\|g\|_{3 / 2}^{3} \leq\|g\|_{1}\|g\|_{2}^{2}
$$

hence

$$
\left(\frac{\|g\|_{3 / 2}}{\|g\|_{2}}\right)^{3} \leq \frac{\|g\|_{1}}{\|g\|_{2}}
$$

which concludes the proof of (4) and so we are done.

\subsection{The formula for $\{0,1\}$ valued functions}

For $\{0,1\}$ valued functions, Theorem 2.1 has a very simple formulation.

Lemma 5.5. Let $f: \prod_{i=1}^{n} \Omega_{i} \rightarrow\{0,1\}$. Then

$$
\left\|\Delta_{i} f\right\|_{1}=2 I_{i}(f) .
$$

Proof. Let $f:[q] \rightarrow\{0,1\}$ be a function with $\mathbf{E}[f]=p$. Note that

$$
\mathbf{E}[|f-p|]=p(1-p)+(1-p) p=2 \operatorname{Var}[f] .
$$

Since

$$
\Delta_{i} f=f-\mathbf{E}\left[f \mid X_{1}, \ldots, X_{i-1}, X_{i+1}, \ldots, X_{n}\right]
$$

we see that

$$
E\left[\mid \Delta_{i}(f) \| X_{1}, \ldots, X_{i-1}, X_{i+1}, \ldots, X_{n}\right]=2 \operatorname{Var}\left[\Delta_{i} \mid X_{1}, \ldots, X_{i-1}, X_{i+1}, X_{n}\right],
$$

and therefore taking expected value of $X_{1}, \ldots, X_{i-1}, X_{i+1}, X_{n}$ we obtain that

$$
\left\|\Delta_{i} f\right\|_{1}=2 I_{i}(f) .
$$

We now obtain the following corollaries 
Corollary 5.6. There exists some universal constant $C$ such that for any probability spaces $(\Omega, \mu)$, and any function $f: \Omega^{n} \rightarrow\{0,1\}$ it holds that

$$
\operatorname{Var}_{\mu}(f) \leq C \log \left(1 / \mu^{*}\right) \sum_{i=1}^{n} \frac{I_{i}(f)}{\log (1 / 2)-\log \left(I_{i}\right) / 2} .
$$

In particular if $I_{i}(f) \leq \delta$ for all $i$ then:

$$
\sum_{i=1}^{n} I_{i}(f) \geq \frac{1}{2 C \log \left(1 / \mu^{*}\right)}(\log (1 / \delta)-\log (1 / 4)) \operatorname{Var}_{\mu}(f) .
$$

Corollary 5.7. There exists some universal constant $C$ such that for any probability space $(\Omega, \mu)$ and any function $f: \Omega^{n} \rightarrow\{0,1\}$ which is symmetric it holds that it holds that

$$
\sum_{i=1}^{n} I_{i}(f) \geq \frac{C}{\log (1 / \alpha)} \log n \operatorname{Var}_{\mu}(f) .
$$

Proof. If $I_{i} \geq \frac{\log n}{100 n}$ the claim follows immediately. Otherwise we apply the previous corollary and note that $\log (1 / \delta)=\Omega(\log n)$.

Remark 5.8. It is a fundamental question both for the Boolean case and for the case of larger alphabet that we consider here to find conditions (and even appropriate definitions) for sharp thresholds for monotone functions. One may want to obtain similar result on the basis of the fact that the function $f$ has low influences. However, this condition on its own does not suffice. Let $m:\{-1,1\}^{2 n} \rightarrow\{-1,1\}$ be the majority function defined as follows:

$$
m_{n}(x)=\operatorname{sign}\left(\sum_{i=1}^{2 n} x_{i}\right)
$$

When the sum is 0 the function is defined arbitrarily in such a way that it is -1 on half of the balanced inputs and 1 on the other half. We write vectors $(x, y) \in\{-1,1\}^{2 n}$ where $x$ and $y$ are two vectors of length $n$. Let $f_{n}=m(x,-y)$. Then clearly all of $f$ influences are of order at most $n^{-1 / 2}$. On the other hand, it is easy to see that $\lim _{n \rightarrow \infty} \mathbf{E}_{p(n)}[f]=0$ for all functions $p(n)$ satisfying $\lim _{n \rightarrow \infty} n p(n)=0$ and $\lim _{n \rightarrow \infty} n(1-p(n))=0$.

\section{References}

[1] J. Bourgain, J. Kahn, G. Kalai, Y. Katznelson, and N. Linial. The influence of variables in product spaces. Israel Journal of Mathematics, 77:55-64, 1992.

[2] P. Dasgupta and E. Maskin. The robustness of majority rule. J. of European Economic Association, 6:949-973, 2008.

[3] B. Efron and C. Stein. The jacknife estimate of variance. Annals of Statistics, 9(3), 1996. 
[4] E. Friedgut and G. Kalai. Every monotone graph property has a sharp threshold. Proc. Amer. Math. Soc., 124:2993-3002, 1996.

[5] G. Kalai. Social Indeterminacy. Econometrica, 72:1565-1581, 2004.

[6] G. A. Margulis. Probabilistic characteristics of graphs with large connectivity. Problemy Peredav ci Informacii, 10(2):101-108, 1974.

[7] D. C. McGarvey. A theorem on the construction of voting paradoxes. Econometrica, 21:608610, 1953.

[8] L. Russo. On the critical percolation probabilities. Z. Wahrsch. Verw. Gebiete, 56(2):229-237, 1981.

[9] D. G. Saari. A dictionary of voting paradoxes. J. Econ. Th., 1989.

[10] M. Talagrand. On Russo's approximate 0-1 law. Annals of Probability, 22:1576-1587, 1994.

[11] P. Wolff. Hypercontractivity of simple random variables. Studia Mathematica, pages 219-326, 2007. 